\title{
Surveillance des brûlures thermiques et des échaudures dans les services d'urgence, d'après la base de données électronique du Système canadien hospitalier d'information et de recherche en prévention des traumatismes, 2013
}

\author{
Jennifer Crain, M.A. (1); Steven McFaull, M. Sc. (1); Deepa P. Rao, Ph. D. (1); Minh T. Do, Ph. D. (1,2); \\ Wendy Thompson, M. Sc. (1)
}

\section{Introduction}

Bien que les taux de mortalité et d'hospitalisation associés à des brûlures au Canada aient diminué avec le temps ${ }^{1,2}$ des cas moins graves se présentent encore couramment aux services d'urgence.

\section{Méthodologie}

Le Système canadien hospitalier d'information et de recherche en prévention des traumatismes (SCHIRPT), un système de surveillance des blessures et des empoisonnements administré par l'Agence de la santé publique du Canada, est en place dans les services d'urgence de 17 hôpitaux ${ }^{3}$. Nous avons fait des recherches dans la base de données électronique du SCHIRPT (eSCHIRPT) afin de connaitre le nombre de personnes de tous âges s'étant présentées aux urgences après avoir subi des brûlures thermiques ou des échaudures en 2013. Les brûlures causées par la friction, par des agents chimiques ou caustiques ou par un contact direct avec la foudre ont été exclues parce qu'il s'agit de circonstances exceptionnelles.

\section{Résultats}

Au total, 1682 cas ont été recensés, soit 1,2 \% (1 682 sur $137245 ; 1226$ pour 100000 cas dans l'eSCHIRPT) des blessures déclarées en 2013. La moitié étaient des échaudures $(52,3 \%$; 879 sur 1 682) et $29,9 \%$ (503 sur 1682 ) résultaient d'un contact avec un objet brûlant (figure 1). Les deux principales causes d'échaudures étaient
FIGURE 1

Distribution des blessures par brûlures selon le mécanisme, fréquence et pourcentage dans tous les groupes d'âge confondus, eSCHIRPT, 2013

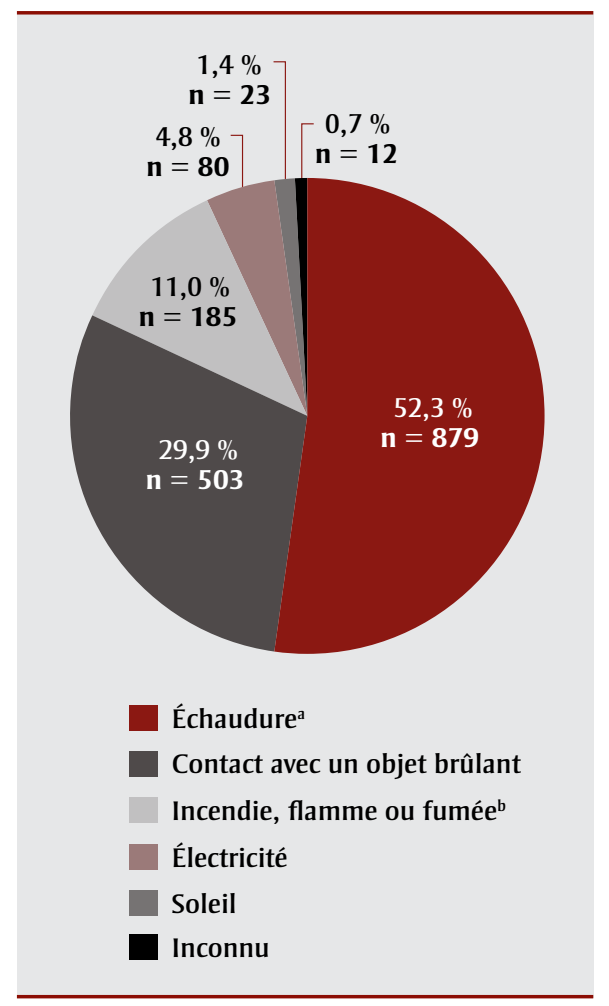

Abréviation : eSCHIRPT, base de données électronique du Système canadien hospitalier d'information et de recherche en prévention des traumatismes.

a Comprend le contact avec l'eau chaude, la vapeur, les aliments, l'huile, la graisse, la colle liquide et la cire liquide.

${ }^{\mathrm{b}}$ Comprend les explosions et le contact avec des braises. les boissons chaudes et l'eau chaude (excluant celle du robinet), avec respectivement $34,1 \%$ (292 sur 856; information manquante dans 23 cas) et $28,9 \%$ (247 sur 856 ; information manquante dans 23 cas) des cas. Les deux principales causes directes de brûlures par contact étaient les cuisinières et fours $(22,0 \%$; 109 sur 495; information manquante dans 8 cas) et les foyers et leurs accessoires (19,6 \%; 97 sur 495; information manquante dans 8 cas). Dans l'ensemble, 13,0 \% des cas (218 sur 1 682) étaient assez graves pour nécessiter une hospitalisation, et la plus forte proportion d'hospitalisations a été enregistrée parmi les personnes exposées à un incendie, à des flammes ou à de la fumée, soit 38,9 \% (72 sur 185).

Si la proportion globale de brûlures était plus élevée chez les femmes, la proportion de brûlures autres que par échaudures était plus élevée chez les hommes (tableau 1).

\section{TABLEAU 1}

Distribution des brûlures selon le mécanisme et le sexe, proportion pour 100000 enregistrements ${ }^{\mathrm{a}}, \mathrm{eSCHIRPT}, 2013$

\begin{tabular}{lrr}
\multicolumn{1}{c}{ Mécanisme thermique } & Hommes & Femmes \\
\hline Échaudure & 544,7 & 767,4 \\
\hline Objet brûlant & 393,5 & 331,3 \\
\hline Incendie, flamme ou fumée ${ }^{\mathrm{b}}$ & 169,2 & 89,6 \\
\hline Électricité $^{2}$ & 71,8 & 40,6 \\
\hline Soleil & 20,5 & 11,8 \\
\hline Inconnu & 7,7 & 10,1 \\
\hline Total & 1207,4 & 1250,8 \\
\hline
\end{tabular}

Abréviation : eSCHIRPT, base de données électronique du Système canadien hospitalier d'information et de recherche en prévention des traumatismes.

a Pour 100000 cas de blessures de tous types chez les personnes de même sexe dans l'eSCHIRPT.

${ }^{\mathrm{b}}$ Comprend les explosions et le contact avec des braises. 
Les figures 2 et 3 illustrent la distribution selon l'âge et le sexe respectivement des échaudures et des brûlures par contact. Les jeunes enfants constituaient le principal groupe d'âge touché par ces types de brûlures.

Dans le cas des brûlures provoquées par un incendie, la flamme ou la fumée, la plus forte proportion selon l'âge et le sexe a été enregistrée chez les hommes de 50 à 64 ans ( $\mathrm{n}=16$; 782 pour 100000 cas dans l'eSCHIRPT), alors que les effectifs les plus

\section{FIGURE 2}

Distribution des échaudures ${ }^{\mathrm{a}}$ selon le groupe d'âge et le sexe, fréquence et proportion pour $1000000^{b}$ enregistrements, eSCHIRPT, 2013

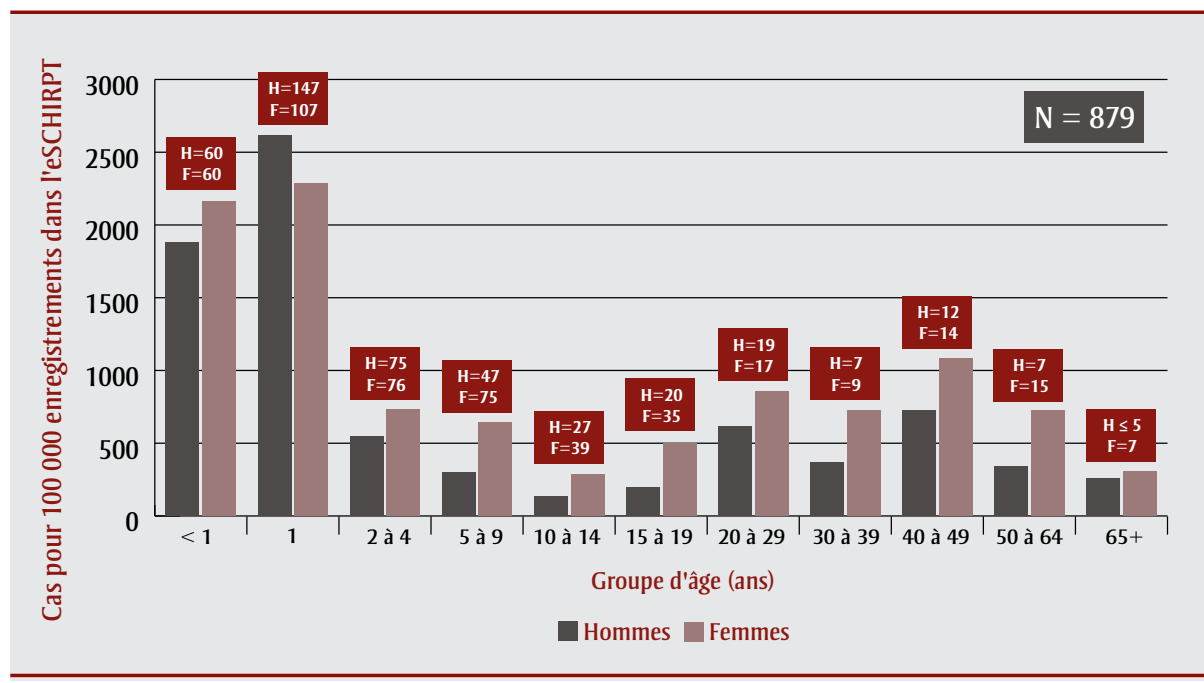

Abréviations : eSCHIRPT, base de données électronique du Système canadien hospitalier d'information et de recherche en prévention des traumatismes; $F$, femmes; $M$, hommes.

Remarque : Les barres colorées représentent la proportion d'hommes et de femmes ayant subi des échaudures pour 100000 cas de blessures de tous types dans l'eSCHIRPT.

${ }^{a}$ Comprend le contact avec l'eau chaude, la vapeur, les aliments, l'huile, la graisse, la colle liquide et la cire liquide.

${ }^{\text {b }}$ Pour 100000 cas de blessures de tous types chez les personnes du même groupe d'âge et du même sexe dans l'eSCHIRPT.

FIGURE 3

Distribution selon le groupe d'âge et le sexe des brûlures par contact avec un objet brûlant, fréquence et proportion pour $100000^{\mathrm{a}}$ enregistrements, eSCHIRPT, 2013

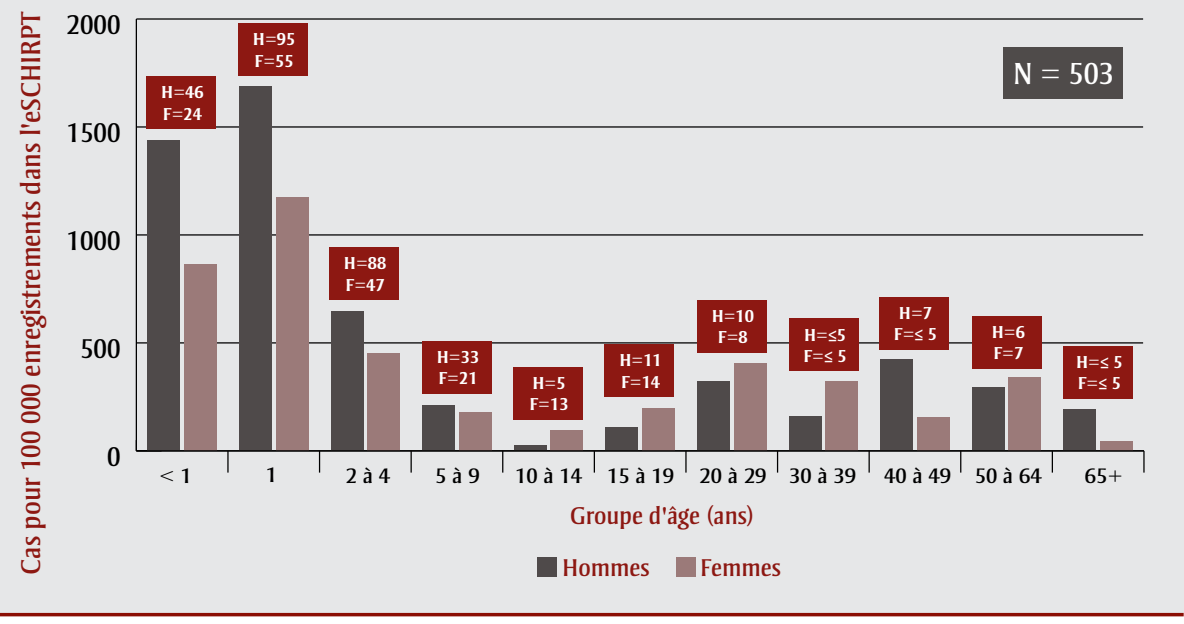

Abréviations : eSCHIRPT, base de données électronique du Système canadien hospitalier d'information et de recherche en prévention des traumatismes; $\mathrm{F}$, femmes; $M$, hommes.

Remarque : Les barres colorées représentent la proportion d'hommes et de femmes ayant subi des échaudures pour 100000 cas de blessures de tous types dans l'eSCHIRPT.

${ }^{a}$ Pour 100000 cas de blessures de tous types chez les personnes du même groupe d'âge et du même sexe dans l'eSCHIRPT. élevés ont été recensés chez les hommes de 15 à 19 ans (n = 21;209,1 pour 100000 cas dans l'eSCHIRPT). La plus forte proportion de brûlures causées par un incendie, la flamme ou la fumée chez les femmes a été répertoriée chez les 20 à 29 ans ( $\mathrm{n}=6$; 302,3 pour 100000 cas dans l'eSCHIRPT).

C'est parmi les hommes et les femmes de 20 à 29 ans que l'on retrouvait les plus fortes proportions de brûlures par le soleil (respectivement 64,8 et 151,1 pour 100000 cas dans l'eSCHIRPT). La proportion de brûlures électriques atteignait son sommet chez les jeunes garçons de moins de 1 an ( $\mathrm{n}=7 ; 219,0$ pour 100000 cas dans l'eSCHIRPT), mais les effectifs les plus élevés $(\mathrm{n}=13)$ ont été enregistrés chez les jeunes garçons de 2 à 4 ans. Les hommes de 20 à 29 ans représentaient la plus forte proportion de cas de brûlures électriques parmi les adultes $(\mathrm{n}=5$; 162,0 pour 100000 cas dans l'eSCHIRPT).

\section{Analyse}

Les brûlures sont omniprésentes dans le SCHIRPT. La forte proportion d'échaudures et de brûlures par contact chez les jeunes enfants semble indiquer la présence de facteurs de risque sociaux et biologiques, notamment une plus grande proportion du temps passé à la maison (où la plupart des brûlures se produisent) et une peau plus jeune et plus mince, donc plus vulnérable aux brûlures. C'est pourquoi il est recommandé de mieux connaître ces facteurs de risque et d'améliorer les mesures de sécurité à adopter.

\section{Limites}

Les résultats en ce qui concerne les mécanismes de brûlure moins courants peuvent être soumis à des variations aléatoires en raison de la petite taille des échantillons. Les cas décrits ne représentent pas non plus tous les cas de brûlures thermiques et d'échaudures au Canada, étant donné que seuls certains hôpitaux participent au SCHIRPT. Tout comme les adolescents de la tranche d'âge supérieure et les adultes, les Autochtones et les habitants des milieux ruraux sont sousreprésentés, car la plupart des établissements qui utilisent le SCHIRPT sont des hôpitaux pour enfants dans les grandes villes. Les cas de décès sont aussi sous-représentés, car les services d'urgences ne saisissent pas les données concernant les personnes qui sont décédées avant leur arrivée à l’hôpital ou après leur admission. 


\section{Références}

1. Statistique Canada. Base de données CANSIM: Tableau 102-0552: Décès et taux de mortalité, selon certains groupes de causes et le sexe, Canada, provinces et territoires, annuel [Internet]. Ottawa (Ont.) : Statistique Canada; [modifié le 9 déc. 2015; cité le 17 août 2016]. En ligne : http:// www5.statcan.gc.ca/cansim/a26?id $=1020552$ \& retrLang $=$ fra\&lang $=$ fra

2. Agence de la santé publique du Canada. Étude des blessures, Édition 2012 : Pleins feux sur la sécurité routière en matière de transport. Ottawa (Ont.) : Agence de la santé publique du Canada; 2012 [no HP1514/2012E-PDF au catalogue].

3. Crain J, McFaull S, Thompson W, Skinner R, Do MT, Fréchette M, Mukhi S. Rapport d'étape : Le Système canadien hospitalier d'information et de recherche en prévention des traumatismes : un système de surveillance des blessures dynamique et novateur. Promotion de la santé et prévention des maladies chroniques au Canada. 2016;36(6):112-117. 